Current Opinion in Neurology - Neuroimaging

\title{
Neuroimaging advances in Parkinson's disease
}

Vittorio Rispoli ${ }^{1,2, *}$, Sebastian R. Schreglmann ${ }^{1, *}$, Kailash P. Bhatia ${ }^{1}$

${ }^{1}$ Sobell Department of Motor Neuroscience and Movement Disorders, Institute of Neurology, UCL, 33

Queen Square, London WC1N 3BG, UK

${ }^{2}$ Department of Neuroscience, University Hospital Arcispedale S. Anna, Aldo Moro street, 8 - Cona, Ferrara -44124, Italy

* contributed equally

\section{Corresponding Author:}

Kailash P. Bhatia' MD, FRCP

${ }^{1}$ Sobell Department of Motor Neuroscience and Movement Disorders

Institute of Neurology, UCL, 33 Queen Square, London WC1N 3BG, UK

k.bhatia@ucl.ac.uk

tel: +442034484229

fax: +44207676 2175

$\begin{array}{lll}\text { word count: } & \text { main } & 2803-2974 / 2500 \\ & \text { abstract } & 183 / 200 \\ & \text { references } & 92 \\ & \text { tables } & 1 \\ & \text { figures } & 3\end{array}$




\section{ABSTRACT}

Purpose of review: Neuroimaging in Parkinson's disease is an evolving field, providing in-vivo insights into the structural and biochemical changes of the condition, although its diagnosis remains clinical. Here, we aim to summarize the most relevant recent advances in neuroimaging in Parkinson's disease to assess the underlying disease process, identify a biomarker of disease progression, and guide or monitor therapeutic interventions.

Recent findings: The clinical applications of imaging technology increasingly allow to quantify pigments (iron, neuromelanin) on MR imaging, proteins (tau), cell markers (phosphodiesterase, microglia) and neurotransmitter receptors (dopamine, serotonin, noradrenalin, cholin) via PET protocols, activity maps by restingstate and task-dependent functional MRI, as well as microstructural changes (free water) through diffusionbased assessments. Their application provides increasing insight on the temporal and spatial dynamics of dopaminergic and other neurotransmitter systems as well as anatomical structures and circuits in PD. An expanding list of PET tracers increases the yield of functional studies.

Summary: This review summarizes the most recent, relevant advances in neuroimaging technology in PD. In particular the combination of different imaging techniques seems promising to maximise the scope of future work, which should, among others, aim at identifying the best imaging marker of disease progression.

key words: $\quad$ PET, SPECT, MRI, imaging, Parkinson's disease 


\section{INTRODUCTION}

Parkinson's disease (PD) is the second most common neurodegenerative disorder after Alzheimer`s dementia[1,2]. Initially perceived as a condition affecting the motor system by $\alpha$-synuclein-mediated loss of dopaminergic neurons in the substantia nigra (SN)[1], current knowledge about associated cognitive, neuropsychiatric and non-motor symptoms has led to increased interest in cholinergic, serotoninergic, noradrenergic and other neurotransmitter systems[3,4]. For some time advanced neuroimaging has been an invaluable tool to investigate in-vivo changes in PD[5]. But while voxel-based morphometry MRI has previously detected volume changes in cortical and subcortical areas[6], state-of the-art neuroimaging nowadays provides a window into the (micro-) structure and function of neuronal networks and surrounding glia [7, 8*].

The aim of this review therefore is to summarize the most relevant recent developments in both structural and functional neuroimaging in PD pertaining to certain aspects: (1) the detection of underpinning neurobiological processes, (2) the use of different neuroimaging techniques as biomarkers of disease progression, and (3) the application of imaging to guide and monitor therapeutic interventions. 


\section{DETECTION OF UNDERLYING NEUROBIOLOGICAL PROCESSES}

Neuroimaging continues to be a powerful tool for the in-vivo study of disease related pathophysiology and recent developments in PD corroborate this.

\section{$\underline{\text { Neurotransmitter systems }}$}

It has been apparent for some time that the degeneration of neurons in PD is not limited to the Substantia nigra, but only recent developments of respective tracers allow the detailed in-vivo study of additional neurotransmitter systems (see Table 1).

\section{Dopaminergic system}

Although quantification of pre-synaptic dopaminergic function is part of daily clinical practice[9], the exact anatomical pattern and longitudinal dynamics of signal changes are increasingly described: a substantial meta-analysis of 142 studies using PET or SPECT to assess presynaptic dopaminergic function confirmed consistently larger decreases in binding in posterior > anterior putamen > caudate nucleus $(\mathrm{CN})$ across ligands for aromatic L-amino-acid decarboxylase (AADC), dopamine transporter (DaT) and vesicular monoamine transporter 2 (VMAT2) - overall disease severity correlated closest with dopaminergic terminal loss in the $\mathrm{CN}\left[10^{* *}, 11\right]$. Focusing on the physiological equivalent of SPECT signalling, a large study reported postmortem numbers of nigral neurons in PD and atypical parkinsonian conditions not to correlate with [ $\left.{ }^{123} \mathrm{I}\right] \mathrm{FP}-$ CIT or $\left[{ }^{123}\right.$ I] b-CIT tracer binding[12*], which contrasts with previous observations[13]. This suggests that these tracers actually reflect striatal changes such as axonal dysfunction or reduced DaT expression, rather than the number of intact nigral neurons.

Similarly, the dynamics of changes among tracer targets are increasingly studied: a substantial meta-analysis reported a consistently smaller reduction in 6-[ $\left[{ }^{18} \mathrm{~F}\right]$ fluoro-L-dopa measuring AADC in both $\mathrm{CN}$ and putamen compared to DaT and VMAT2 levels, suggesting either less severe loss or even compensatory up-regulation of AADC in PD[10**]. An analysis of a large number $(n=210)$ of healthy control DaTSCANs indicated a relatively higher DaT concentration in women, as female striatal volumes are slightly lower, but DaT quantification similar between sexes[14]. 
Nigro-striatal dopaminergic loss as quantified by DaT, has also been used to study pathophysiological changes only accessible to advanced imaging techniques by means of correlation analysis: one study on the basis of diffusion tensor imaging (DTI) reported a negative correlation between fractional anisotropy of the SN and putaminal dopamine transporter signal over time[15*], while another cross-sectional study described that on neuromelanin-sensitive sequences, SN volume correlated best with nigro-striatal innervation loss[16]. Both studies detected micro-structural changes based on correlations with established DaT quantification. Similarly, the severity of dopaminergic degeneration correlated with changes in diffusion measurements in the striatum in a primate MPTP model[17].

Today, the in-vivo study of dopaminergic degeneration is able to detect the temporal and spatial dynamics of tracer changes and serves as a basis for the explorative study of novel imaging approaches.

\section{Serotoninergic system}

Although serotoninergic dysfunction is known to occur in PD, in-vivo quantification has been achieved only recently. $\left[{ }^{123} \mathrm{I}\right] \mathrm{FP}-\mathrm{CIT}$ exhibits non-specific, extrastriatal binding for presynaptic serotonergic transporters[18,19*] and hence has been used to assess its involvement in the pathogenesis of non-motor symptoms. One study found significant differences in hypothalamic binding between PD and atypical parkinsonism, suggesting a different pattern of serotonergic pre-synaptic receptor involvement between these conditions[20]. More specifically, the same team reported a negative association of levels of anxiety with low [ ${ }^{123}$ I]FP-CIT binding in the thalamus in a large sample of de novo PD patients[19*]. A similar negative correlation between right $\mathrm{CN}\left[{ }^{123} \mathrm{I}\right] \mathrm{FP}-\mathrm{CIT}$ SPECT tracer binding and anxiety has been reported in a large, controlled cohort of de novo PD patients[21], confirming an earlier observation[22].

Although the above mentioned results are in line with the hypothesis of anxiety mainly being linked to a serotoninergic dysfunction in earlier and a dopaminergic dysfunction in later disease stages[20], this remains to be proven in larger, and ideally longitudinal samples. 


\section{Noradrenergic system}

Noradrenergic projections almost exclusively originate from the locus coeruleus (LC), playing a role in cognitive attention, sleep cycle regulation and autonomic function. The application of neuromelanin-sensitive MRI sequences to identify pigmented cells of the LC $\left[23^{*}\right]$ and $\left[{ }^{11} \mathrm{C}\right]$ MeNER PET to characterize noradrenalin transporters[24] allowed a study comparing PD patients with and without rapid eye movement sleep behaviour disorder (RBD) and controls, revealing most pronounced reductions for both signals in the PD+RBD group[23*], supporting an earlier neuromelanin-based study[25] (see Figure 1). In terms of dynamics, first evidence indicates that the reduction in neuromelanin-content might be greater in the LC than SN [16], possibly reflecting the progression of neuropathology according to the Braak hypothesis[27]. Although of minuscule anatomical size, the noradrenergic system and the implications of changes to it, is now accessible to state-of the art imaging.

\section{Cholinergic system}

There has been a debate over the anatomical and functional aetiology of cognitive symptoms in PD, which present most frequently as executive, and in later stages more wide-spread dysfunction including memory impairment[28]. A noticeable PET-based contribution is the finding that it might be the interaction between $\mathrm{CN}$ dopaminergic and cortical cholinergic denervation to explain PD cognitive decline and dementia[29,30**]. The data seems to suggest a possible compensatory over-activity of the cortical cholinergic system for $\mathrm{CN}$ dopaminergic degeneration in early disease stages, before cognitive symptoms become apparent with cholinergic decline. This challenges the previously proposed dual syndrome hypothesis, which distinguishes dopaminergically mediated fronto-striatal executive impairments and cholinergically mediated memory symptoms[28]. 


\section{$\underline{\text { Protein aggregates }}$}

PD neuropathology is dominated by the presence of $\alpha$-synuclein deposits, although $\beta$-amyloid and tau have been implicated in cognitive impairment in $\mathrm{PD}\left[31^{*}, 32^{*}\right]$. Recent publications seem to support a role for both tau and $\beta$-amyloid only in advanced stages of cognitive impairment [31*, 32*, 33], most noticeably in dementia with lewy bodies[31*]. Anatomically, precuneus and inferior temporal gyrus tau tracer uptake has been reported to be associated with this [34], adding to our understanding of mixed pathology in PD.

\section{White matter involvement}

Overt white matter (WM) lesions have been implicated with the development of both cognitive symptoms and gait disorder in PD. The reported observation of an association between WM lesions and neurogenic orthostatic hypotension[35] supports the notion that extreme blood pressure changes might contribute to the development of cognitive symptoms through WM lesions[36]. Small vessel involvement, defined as lacunes or WM hyperintensities, have equally been linked to cortical thinning and cognitive impairment[37]. Greater WM lesion load is reported among patients presenting with postural instability and gait difficulty compared to a tremor-dominant phenotype[3,18].

Furthermore, computational and theoretical advances allow for a more comprehensive assessment of microstructural changes and network disruptions: A meta-analysis of 39 DTI studies comparing data from 1087 PD patients and 768 healthy controls identified consistent changes within subcortical (SN) and cortical areas (cingulate and temporal cortices) as well as white matter fibre tracts (corpus callosum and cortico-spinal tract)[7]. Prospective high-resolution 3 Tesla DTI imaging and graph theory detected reduced connectivity in mesolimbic-striatal pathways, within the basal ganglia, the sensorimotor circuits and also the limbic system in a cohort of treatment naïve patients[11]. Another study suggested more prominent WM lesions along the cerebello-thalamo-cortical pathway in tremor-dominant PD patients[17]. A large study of advanced PD patients also identified DTI changes in diffuse basal ganglia and frontoparietal networks among PD patients with mild cognitive impairment[24]. Taken together, these findings point towards an early, progressive degeneration initially evident only at a microstructure level[25].

3. Wen MC, Heng HSE, Lu Z, et al. (2018) Differential White Matter Regional Alterations in Motor 
Subtypes of Early Drug-Naive Parkinson's Disease Patients. Neurorehabil Neural Repair. doi: $10.1177 / 1545968317753075$

18. Al-Bachari S, Vidyasagar R, Emsley HC, Parkes LM (2017) Structural and physiological neurovascular changes in idiopathic Parkinson's disease and its clinical phenotypes. J Cereb Blood Flow Metab 37:3409-3421. doi: 10.1177/0271678X16688919

7. Atkinson-Clement C, Pinto S, Eusebio A, Coulon O (2017) Diffusion tensor imaging in Parkinson's disease: Review and meta-analysis. NeuroImage Clin 16:98-110. doi: 10.1016/j.nicl.2017.07.011

11. Nigro S, Riccelli R, Passamonti L, et al. (2016) Characterizing structural neural networks in de novo Parkinson disease patients using diffusion tensor imaging. Hum Brain Mapp 37:4500-4510. doi: $10.1002 / \mathrm{hbm} .23324$

24. Galantucci S, Agosta F, Stefanova E, et al. (2017) Structural Brain Connectome and Cognitive Impairment in Parkinson Disease. Radiology 283:515-525. doi: 10.1148/radiol.2016160274

17. Luo C, Song W, Chen Q, et al. (2017) White matter microstructure damage in tremor-dominant Parkinson's disease patients. Neuroradiology 59:691-698. doi: 10.1007/s00234-017-1846-7

\section{$\underline{\text { Neuroinflammation }}$}

A number of findings suggest a role of the immune system in PD pathoaetiology[1]. Activation of microglia may be evaluated through novel PET radioligands, such as $\left[{ }^{18} \mathrm{~F}\right] \mathrm{FEPPA}$, although first, moderately sized studies in PD were not able to show signal differences in comparison to healthy controls [38,39]. $\left[{ }^{11} \mathrm{C}\right] \mathrm{PBR} 28$ imaging, however, revealed a reduction in microglia activation after treatment with a selective myeloperoxidase inhibitor in PD although this report included no control subjects for comparison[40]. Indirect indications of inflammation have also been detected by signs of blood brain barrier breakdown, evident as microbleeds and gadolinium leakage[41].

\section{Phosphodiesterases}

Phosphodiesterases are enzymes highly expressed on striatal medium spiny neurons, metabolizing cAMP and cGMP, the second messengers of dopamine receptors. First studies employing specific radioligands, such as $\left[{ }^{11} \mathrm{C}\right] \mathrm{IMA} 107$ binding to phosphodiesterase 10A[42], showed lower binding activity in the basal ganglia of PD patients compared to controls, and a negative correlation with disease duration and severity[43]. The same group adopted $\left[{ }^{11} \mathrm{C}\right]$ rolipram, assessing phosphodiesterase 4 levels, and reported lower binding in 
caudate, putamen, hypothalamus and cortex to correlate with spatial working memory deterioration[44**]. Positing phosphodiesterase expression as a marker of striatal dopaminergic terminal loss, it would be interesting to study its decline longitudinally and compare its dynamics with DaT and other markers of dopaminergic degeneration in the future.

\section{$\underline{\text { Functional MRI }}$}

The application of functional MRI (fMRI) studies in PD is still an evolving field, with a considerable heterogeneity of paradigms and patient subgroups being studied. Nevertheless, a methodologically sound, recent meta-analysis of resting state fMRI studies indicated reproducible, increased functional connectivity in the left postcentral gyrus[45]. While this might highlight this area to be explored further,, the conclusive interpretation of results of task-dependent fMRI studies[46] remains challenging, still.

\section{IMAGING MODALITIES AS BIOMARKER OF DISEASE PROGRESSION}

Monitoring disease progression is a prime aim in PD imaging research, but although there have been some developments, it remains to be seen which modality might emerge as the most exact measure.

\section{$\underline{\text { Iron-content }}$}

Brain tissues physiologically contains iron, $>90 \%$ of which is stored in the form of ferritin, and the basal ganglia (Globus Pallidus (GP) > Putamen > Caudate) are particularly rich in iron. Imaging studies have shown that ferritin accumulates throughout the basal ganglia in a linear fashion as a function of age[47]. Iron-sensitive MRI sequences including SWI, 3D FLAIR, T2*, R2 and R2* relaxation, as well as quantitative susceptibility mapping (QSM) or adiabatic T1rho have been increasingly applied to study iron-content and -metabolism in PD[48]. Overall, QSM has been suggested to be more sensitive than R2* to detect deep cerebral nuclei iron quantity[48,49], allowing the visualization of the dorsolateral nigral hyperintesity or nigrosome-1 within the SN. A meta-analysis of 16 studies suggested this assessment to be the most sensitive and specific imaging protocol to date to differentiate PD patients from controls and possible suitable to differentiate degenerative from non-degenerative parkinsonism[50*]. Nigral hyperintensity has also been corre- 
lated with disease progression and reported to predict DaTSCAN measures in PD[51,52], but also idiopathic $\operatorname{RBD}[53]$.

Disease severity, as measured by clinical characteristics ranging from UPDRS-III, H\&Y stage, depression and anxiety to levodopa equivalent daily dose, has been reported to correlate with iron load in $\mathrm{SN}$ on SWI[54*], R2* sequences[55], and QSM[48,56]. Iron load, especially in the caudal region of the SN (R2*) has been reported to be particularly higher in patients with freezing of gait in both cross-sectional[49] and longitudinal samples[55]. Taken together, as an epiphenomenon of the underpinning neuropathological process, iron accumulation seems to be a good candidate to follow disease progression in PD.

Regarding extranigral tissue in PD in comparison to controls, iron load was found increased in GP and putamen on SWI-weighted sequences[54*], and red nucleus (RN)[48], GP[48] and thalamus[48] on QSM quantification[48]. A QSM-based study described iron signal changes only in SNc in early disease stages, whereas more prominent involvement of RN and GP were apparent in more advanced patients[57]. Only one study reported whole brain iron level assessments - using a cross-sectional protocol it reported higher QSM values in dorsal pons and a number of cortical areas, arguably following the topographical progression of $\alpha$ synuclein deposition[58].

Iron content therefore seems to emerge as a powerful imaging tool with high diagnostic accuracy (nigral hyperintensity), good correlation with clinical characteristics and good spatial as well as longitudinal temporal resolution.

\section{Neuromelanin}

Neuromelanin is an insoluble pigment and by-product of catecholamine-synthesis. Its production is $\mathrm{Fe} 3+-$ mediated and appears to be governed by cytosolic content of dopamine or other catecholamines and is therefore predominantly located in SN and locus coeruleus. Quantification of neuromelanin to follow disease pro- 
gression has been investigated widely. The histologically known pattern of SN neuronal changes affecting postero-lateral aspects of SNc earlier/more than anterior-medial ones has been replicated with neuromelanin imaging[59-61]. Similarly, the progression of loss of SWI-hyperintensity from nigrosome-1 to nigrosome-4 seems to follow disease progression[62]. Neuromelanin loss has also been correlated with UPDRS[59,60] and MDS-UPDRS scores[61], H\&Y stages[61] and disease duration[63].

Hence, neuromelanin appears to be a promising imaging biomarker for PD progression, if confirmed prospectively.

\section{Substantia nigra Free Water}

Free water measurements are an advanced diffusion-based modelling approach, measuring the fractional volume of unconstrained diffusion. The recent development of a bitensor modelling technique allows to acquire the fractional signal of water from a single voxel. The detection of posterior $\mathrm{SN}$ free water was able to distinguish PD patients from controls[64,65**] and in a multisite study showed to correlate with H\&Y stages, MDS-UPDRS part III and cognitive performance [66]. This appeared to be independent of acute dopaminergic medication[67]. First longitudinal data indicated that posterior SN free water increases over time and can predict bradykinesia severity and MOCA scores[68], which was validated in a larger, international study $\left[65^{* *}\right]$ (see Figure 2). By and large, all of the above suggests that SN free water might become a valuable tool to monitor PD progression.

\section{DaTSCAN}

It is generally accepted that DaT measurements correlate with the degree of dopaminergic cell loss, however its exact dynamics across disease stages has not been clear. A recent study implied different velocities of midbrain neuromelanin-signal loss measured via $\left[{ }^{18} \mathrm{~F}\right] \mathrm{AV}-1451$ PET $\left[69^{*}\right]$, versus striatal dopaminergic loss ([23I]FP-CIT SPECT) in early PD[70]. Together with further evidence[71*], this has been interpreted to indicate that in humans nigral cell loss and striatal tracer uptake in-vivo correlate closely only up to a nigral cell loss of 50\%. Beyond this, the dynamics of neurodegeneration and tracer uptake seem to follow a more lose correlation, as also observed in MPTP-treated monkeys[72]. Accordingly, the majority of longitudinal 
PET studies[10**] point to a negative exponential dopaminergic loss in PD, consistent with the nonlinear pattern previously described for the progression of motor symptoms, with faster progression in the first symptomatic disease phase[10**]. This is in keeping with the „dying back“ concept, describing axonal and terminal degeneration in the striatum before neuronal cell loss in SN[73]).

\section{GUIDING AND MONITORING THERAPEUTIC INTERVENTIONS}

We have noted some developments in the application of imaging used for therapeutic interventions in PD.

\section{Functional neurosurgery}

Both deep brain stimulating and advanced lesioning[74] rely on the correct pre-surgical identification of target structures. One report proposed STN visualization to benefit from using QSM over T2*-weighted images[75] (see Figure 3). Similarly, advanced high angular resolution diffusion-based imaging has been proposed to improve VIM nucleus targeting in tremor patients[76*]. Probably the most innovative addition to functional stereotactic neurosurgery has been the development of the publicly available "DBS Intrinsic Template Atlas" $\left[77^{* *}\right]$ - this combination of subcortical brain manual segmentation, automatic multimodal probabilistic maps, co-registration with histology as well as STN and GP internus functional subsegmentation according to structural connectivity, offers potential advantages for future functional neurosurgical trials[77**].

Another set of studies investigated the effect of both anatomical and functional connectivity after STN-DBS and validated the findings in a second, independent data set[78**]: apart from replicating that STN-DBS has an effect on SMA and medial prefrontal cortex activity, authors reported that both types of connectivity explained $26.2 \%$ of clinical variance in intervention outcome. They concluded that their findings support the idea of focusing surgical treatment not only on specific brain regions, but also on network nodes[78**].

Finally, of more practical relevance, the development of a new coil system able to reduce DBS lead absorption might alleviate safety concerns for post-DBS MRI monitoring[79].

\section{$\underline{\text { Levodopa/carbidopa intestinal gel (LCIG) }}$}


Continuous delivery of levodopa via LCIG provides more stable levodopa levels in blood with respective clinical advantages over pulsatile oral intake, which is advantageous in advanced PD cases. A small pilot study documented a sustained significant reduction in striatal $\left[{ }^{11} \mathrm{C}\right]$ raclopride binding, suggesting sustained higher dopamine levels during LCIG infusion[80].

\section{$\underline{\text { Apomorphine }}$}

One study examined the effect of apomorphine infusions as add-on therapy using resting state PET over a 6 month period: induced changes in this relatively small sample $(n=12)$ were documented throughout motor, cognitive and limbic networks and appear compatible with the notion that metabolic changes in PD could be reversed by continuous dopaminergic treatment [81].

\section{$\underline{\text { Rehabilitation }}$}

A randomized trial of cognitive rehabilitative approaches documented improved executive function, visual memory and functional but no structural changes on functional MRI after three months of integrative cognitive rehabilitation[82*], persisting at 18 months[83].

\section{SUMMARY}

In this review we provide an update on current applications of state of the art neuroimaging in PD. The expansion of the current in-vivo imaging armamentarium increasingly allows the comparison and crossvalidation of different imaging modalities. It seems that the increasing number of radionuclide-based proto- 
cols assessing microglia activation, protein accumulation and receptor density as well as the combination of established and novel approaches drive developments in this field of movement disorder research. Providing distinct and complementary information, in particular the combination of these techniques will most likely advance our understanding of the pathophysiology of PD disease processes in the future. Among other, future work should focus on identifying the best marker to monitor disease progression.

\section{KEY POINTS:}

- The increasing number of available PET tracers marking neurotransmitters, proteins and cell populations dramatically increases the scope for future studies.

- Iron content, neuromelanin and substantia nigra free water content seem to emerge as potential markers of disease progression along conventional DaT scans.

- The combination of different imaging modalities is increasingly applied and allows deeper insights into the pathophysiology of the condition..

\section{Acknowledgements:}

none 
This work has received no dedicated support.

\section{Conflicts of interest:}

VR declares received travel grants from AbbVie, Boston Scientific and Italian Neurological Society. SRS has received grant support by the Swiss Science Foundation, the Swiss Neurological Society and the European Academy of Neurology. KPB has received grant support from welcome/MRC, NIHR, Parkinson's UK and EU horizon 2020. He has received royalties from publication of the Oxford Specialist Handbook Parkinson's Disease and Other Movement Disorders (Oxford University Press, 2008, 2016) and of Marsden's Book of Movement Disorders (Oxford University Press, 2013). He has received honoraria/personal compensation for participating as consultant/scientific board member from Ipsen, Allergan, Merz and honoraria for speaking at meetings and from Allergan, Ipsen, Merz, Sun Pharma, Teva, UCB Pharmaceuticals and from the American Academy of Neurology and movement disorders society.

ABBREVIATIONS:

\begin{tabular}{c|c} 
AADC & aromatic L-amino-acid decarboxylase \\
\hline CN & caudate nucleus \\
\hline DaT & dopamine transporter \\
\hline DBS & deep brain stimulation \\
\hline
\end{tabular}




\begin{tabular}{c|c} 
DTI & diffusion tensor imaging \\
\hline GM & $\begin{array}{c}\text { grey matter } \\
\text { globus pallidus }\end{array}$ \\
\hline LC & locus coeruleus \\
\hline LCIG & levodopa/carbidopa intestinal gel \\
\hline MRI & magnetic resonance imaging \\
\hline PET & positron emission tomography \\
\hline QSM & quantitative susceptibility mapping \\
\hline RBD & rapid eye movement sleep behaviour disorder \\
\hline RN & substantia nigra \\
\hline SN & subthalamic nucleus \\
\hline SPECT & susceptibility weighted imaging \\
\hline STN & ventral intermediate nucleus of thalamus \\
\hline SWI & vesicular monoamine transporter 2 \\
\hline VIM & white matter
\end{tabular}

\section{REFERENCES:}

1. Poewe W, Seppi K, Tanner CM, et al.: Parkinson disease. Nat Rev Dis Prim 2017, 3:17013. 
2. Hirtz D, Thurman DJ, Gwinn-Hardy K, et al.: How common are the 'common' neurologic disorders? Neurology 2007, 68:326-337.

3. Titova N, Padmakumar C, Lewis SJG, et al.: Parkinson's: a syndrome rather than a disease? J Neural Transm 2017, 124:907-914.

4. Titova N, Qamar MA, Chaudhuri KR: Biomarkers of Parkinson's Disease: An Introduction. In International Review of Neurobiology. . 2017:183-196.

5. Politis M: Neuroimaging in Parkinson disease: from research setting to clinical practice. Nat Rev Neurol 2014, 10:708-722.

6. Jia X, Liang P, Li Y, et al.: Longitudinal Study of Gray Matter Changes in Parkinson Disease. Am J Neuroradiol 2015, 36:2219-2226.

7. Saeed U, Compagnone J, Aviv RI, et al.: Imaging biomarkers in Parkinson's disease and Parkinsonian syndromes: current and emerging concepts. Transl Neurodegener 2017, 6:8.

* 8. Strafella AP, Bohnen NI, Perlmutter JS, et al.: Molecular imaging to track Parkinson's disease and atypical parkinsonisms: New imaging frontiers. Mov Disord 2017, 32:181192.

It is an outstanding clear review on molecular imaging, focused on cognitive impairment and psychiatric complications in PD.

9. Kagi G, Bhatia KP, Tolosa E: The role of DAT-SPECT in movement disorders. J Neurol Neurosurg Psychiatry 2010, 81:5-12.

** 10. Kaasinen V, Vahlberg T: Striatal dopamine in Parkinson disease: A meta-analysis of imaging studies. Ann Neurol 2017, 82:873-882.

This is the first comprehensive meta-analysis on PET and SPECT studies analysing the dopaminergic denervation in PD. Importanly, it pointed out an exponenatial loss of nigral dopaminergic projections and the relation across AADC, VMAT2, and DAT tracer-related findings.

11. Prashanth R, Dutta Roy S, Mandal PK, et al.: High-Accuracy Classification of Parkinson's Disease Through Shape Analysis and Surface Fitting in 123I-Ioflupane SPECT Imaging. IEEE J Biomed Heal Informatics 2017, 21:794-802.

* 12. Saari L, Kivinen K, Gardberg M, et al.: Dopamine transporter imaging does not predict the number of nigral neurons in Parkinson disease. Neurology 2017, 88:1461-1467.

Despite the larger employment of SPECT to assess the presence of DaT degeneration, it is not clear whether the results of this test reflect neuropathological correlates of PD. This is the sole study to address this question in a small sample of PD patients.

13. Kraemmer J, Kovacs GG, Perju-Dumbrava L, et al.: Correlation of striatal dopamine transporter imaging with post mortem substantia nigra cell counts. Mov Disord 2014, 29:1767-1773.

14. Tagare HD, DeLorenzo C, Chelikani S, et al.: Voxel-based logistic analysis of PPMI control and Parkinson's disease DaTscans. Neuroimage 2017, 152:299-311.

* 15. Zhang Y, Wu I-W, Tosun D, et al.: Progression of Regional Microstructural Degeneration in Parkinson's Disease: A Multicenter Diffusion Tensor Imaging Study. PLoS One 2016, 11:e0165540.

This paper describes the SN microstructural alterations on a large cohort of PD patients and controls from the PPMI database. Apart from the DTI change annual rate, the authors correlated for the first time this parameter to DaTs, as measured by $\left[{ }^{123} \mathrm{I}\right] \mathrm{FP}-\mathrm{CIT}$, and to CSF concentration of $\alpha$-synuclein and tau protein.

16. Isaias IU, Trujillo $P$, Summers $P$, et al.: Neuromelanin Imaging and Dopaminergic Loss in Parkinson's Disease. Front Aging Neurosci 2016, 8:1-12.

17. Météreau E, Beaudoin-Gobert M, Duperrier S, et al.: Diffusion tensor imaging marks dopaminergic and serotonergic lesions in the Parkinsonian monkey. Mov Disord 2018, 33:298-309. 
18. Jaakkola E, Joutsa J, Mäkinen E, et al.: Ventral striatal dopaminergic defect is associated with hallucinations in Parkinson's disease. Eur J Neurol 2017, 24:1341-1347.

* 19. Joling M, van den Heuvel OA, Berendse HW, et al.: Serotonin transporter binding and anxiety symptoms in Parkinson's disease. J Neurol Neurosurg Psychiatry 2018, 89:89-94.

The novelty of this paper is in pointing to a thalamic serotonergic transporter involvement in PD anxiety, as measured by the $\left[{ }^{123} \mathrm{I}\right] \mathrm{FP}-\mathrm{CIT}$ extra-strital binding.

,20. Joling M, Vriend C, van den Heuvel OA, et al.: Analysis of Extrastriatal 123 I-FP-CIT Binding Contributes to the Differential Diagnosis of Parkinsonian Diseases. $\mathrm{J} \mathrm{Nucl} \mathrm{Med}$ 2017, 58:1117-1123.

21. Picillo M, Santangelo G, Erro R, et al.: Association between dopaminergic dysfunction and anxiety in de novo Parkinson's disease. Parkinsonism Relat Disord 2017, 37:106-110.

22. Erro R, Pappatà S, Amboni M, et al.: Anxiety is associated with striatal dopamine transporter availability in newly diagnosed untreated Parkinson's disease patients. Parkinsonism Relat Disord 2012, 18:1034-1038.

* 23. Sommerauer M, Fedorova TD, Hansen AK, et al.: Evaluation of the noradrenergic system in Parkinson's disease: an 11C-MeNER PET and neuromelanin MRI study. Brain 2018, 141:496-504.

This study evaluates in-vivo for the first time the noradrenegic degeneration in PD with the means of neuromelanin-sensitive MRI sequences and ${ }^{11} \mathrm{C}$-MeNER: they traced a link between neuradrenergic loss (mainly in locus coeruleus) and three of the most common non-motor symptoms (RBD, orthostatic hypotention and cognitive decline).

24. Nahimi A, Sommerauer M, Kinnerup MB, et al.: Noradrenergic Deficits in Parkinson Disease Imaged with 11 C-MeNER. J Nucl Med 2018, 59:659-664.

25. García-Lorenzo D, Longo-Dos Santos C, Ewenczyk C, et al.: The coeruleus/subcoeruleus complex in rapid eye movement sleep behaviour disorders in Parkinson's disease. Brain 2013, 136:2120-2129.

26. Fereshtehnejad S-M, Romenets SR, Anang JBM, et al.: New Clinical Subtypes of Parkinson Disease and Their Longitudinal Progression. JAMA Neurol 2015, 72:863.

27. Braak H, Rüb U, Gai WP, et al.: Idiopathic Parkinson's disease: possible routes by which vulnerable neuronal types may be subject to neuroinvasion by an unknown pathogen. $J$ Neural Transm 2003, 110:517-536.

28. Kehagia AA, Barker RA, Robbins TW: Cognitive impairment in Parkinson's disease: The Dual Syndrome Hypothesis. Neurodegener Dis 2013, 11:79-92.

29. Kim K, Bohnen NI, Müller MLTM, et al.: Compensatory dopaminergic-cholinergic interactions in conflict processing: Evidence from patients with Parkinson's disease. Neuroimage 2018, doi:10.1016/j.neuroimage.2018.01.021.

** 30. Bohnen NI, Albin RL, Müller MLTM, et al.: Frequency of Cholinergic and Caudate Nucleus Dopaminergic Deficits Across the Predemented Cognitive Spectrum of Parkinson Disease and Evidence of Interaction Effects. JAMA Neurol 2015, 72:194.

This paper suggestes the relation between dopaminergic and cholinergic systems in PD cognitive impairment pathophysiology: in particular, the degeneration of former sparks the hyperacitivity of the latter as a compensatory mechanism, after the failure of which more prominent cognitive symptoms become apparent.

* 31. Petrou M, Dwamena BA, Foerster BR, et al.: Amyloid deposition in Parkinson's disease and cognitive impairment: A systematic review. Mov Disord 2015, 30:928-935.

This systematic review summarized PiB-PET findindings on $\beta$-amyloid, since previous studies relied on small sample and provided not consistent results.

* 32. Winer JR, Maass A, Pressman P, et al.: Associations Between Tau, $\beta$-Amyloid, and Cognition in Parkinson Disease. JAMA Neurol 2018, 75:227.

In this study authors described the first combined approach consisting in Tau and $\beta$-Amyloidbinding tracers to study PD cognitive impairment: tau protein deposition seemed to be related 
to $\beta$-Amyloid and age of patients, but not to cognitive performaces per se.

33. Akhtar RS, Xie SX, Chen YJ, et al.: Regional brain amyloid- $\beta$ accumulation associates with domain-specific cognitive performance in Parkinson disease without dementia. PLoS One 2017, 12:e177924.

34. Gomperts SN, Locascio JJ, Makaretz SJ, Set al.: Tau Positron Emission Tomographic Imaging in the Lewy Body Diseases. JAMA Neurol 2016, 73:1334.

35. ten Harmsen BL, van Rumund A, Aerts MB, et al.: Clinical correlates of cerebral white matter abnormalities in patients with Parkinson's disease. Parkinsonism Relat Disord 2018, 49:28-33.

36. Malek N, Lawton MA, Swallow DMA, et al.: Vascular disease and vascular risk factors in relation to motor features and cognition in early Parkinson's disease. Mov Disord 2016, 31:1518-1526.

37. Foo H, Mak E, Yong TT, et al.: Progression of small vessel disease correlates with cortical thinning in Parkinson's disease. Parkinsonism Relat Disord 2016, 31:34-40.

38. Koshimori Y, Ko J-H, Mizrahi R, et al.: Imaging Striatal Microglial Activation in Patients with Parkinson's Disease. PLoS One 2015, 10:e0138721.

39. Ghadery C, Koshimori Y, Coakeley S, et al.: Microglial activation in Parkinson's disease using [18F]-FEPPA. J Neuroinflammation 2017, 14:8.

40. Jucaite A, Svenningsson P, Rinne JO, et al.: Effect of the myeloperoxidase inhibitor AZD3241 on microglia: a PET study in Parkinson's disease. Brain 2015, 138:2687-2700.

41. Sweeney MD, Sagare AP, Zlokovic B V: Blood-brain barrier breakdown in Alzheimer disease and other neurodegenerative disorders. Nat Rev Neurol 2018, 14:133-150.

42. Plisson C, Weinzimmer D, Jakobsen S, et al.: Phosphodiesterase 10A PET Radioligand Development Program: From Pig to Human. J Nucl Med 2014, 55:595-601.

43. Niccolini F, Foltynie T, Reis Marques T, et al.: Loss of phosphodiesterase 10A expression is associated with progression and severity in Parkinson's disease. Brain 2015, 138:3003-3015.

** 44. Niccolini F, Wilson H, Pagano G, et al.: Loss of phosphodiesterase 4 in Parkinson disease. Neurology 2017, 89:586-593.

Niccolini and colleagues, using for the first time $\left[{ }^{11} \mathrm{C}\right]$ rolipram to study phosphodiesterase 4 invivo in PD, attributed spatial working memory scores to the expression of this enzyme in the striato-thalamo-cortical network.

45. Ji G-J, Hu P, Liu T-T, et al.: Functional Connectivity of the Corticobasal GangliaThalamocortical Network in Parkinson Disease: A Systematic Review and MetaAnalysis with Cross-Validation. Radiology 2018, doi:10.1148/radiol.2018172183.

46. Herz DM, Eickhoff SB, Løkkegaard A, et al.: Functional neuroimaging of motor control in parkinson's disease: A meta-analysis. Hum Brain Mapp 2014, 35:3227-3237.

47. Bartzokis G, Tishler TA, Lu PH, et al.: Brain ferritin iron may influence age- and genderrelated risks of neurodegeneration. Neurobiol Aging 2007, 28:414-423.

48. Langkammer C, Pirpamer L, Seiler S, et al:: Quantitative Susceptibility Mapping in Parkinson's Disease. PLoS One 2016, 11:e0162460.

49. Naduthota RM, Honnedevasthana AA, Lenka A, et al.: Association of freezing of gait with nigral iron accumulation in patients with Parkinson's disease. J Neurol Sci 2017, 382:61-65.

* 50. Mahlknecht $\mathrm{P}$, Krismer $\mathrm{F}$, Poewe $\mathrm{W}$, et al.: Meta-analysis of dorsolateral nigral hyperintensity on magnetic resonance imaging as a marker for Parkinson's disease. Mov Disord 2017, 32:619-623.

Using a meta-analysis, authors confirmed the usefulness of nigral iron content, as measured by MRI, to differentiate PD from controls and other types of parkinsonism not related to neurodegenerations and its correlation with DaT analysis.

51. Oh SW, Shin N-Y, Lee JJ, et al.: Correlation of 3D FLAIR and Dopamine Transporter 
Imaging in Patients With Parkinsonism. Am J Roentgenol 2016, 207:1089-1094.

52. Bae YJ, Kim JM, Kim E, et al.: Loss of Nigral Hyperintensity on 3 Tesla MRI of Parkinsonism: Comparison With ${ }^{123}$ I-FP-CIT SPECT. Mov Disord 2016, 31:684-692.

53. Bae YJ, Kim J-M, Kim KJ, et al.: Loss of Substantia Nigra Hyperintensity at 3.0-T MR Imaging in Idiopathic REM Sleep Behavior Disorder: Comparison with 123 I-FP-CIT SPECT. Radiology 2018, 287:285-293.

* 54. Martin-Bastida A, Lao-Kaim NP, Loane C, et al.: Motor associations of iron accumulation in deep grey matter nuclei in Parkinson's disease: a cross-sectional study of iron-related magnetic resonance imaging susceptibility. Eur J Neurol 2017, 24:357-365.

The SN iron quantification by high-pass filtered phase of SWI RMI was suggested as a measure of PD progression as it correlated with disease severity.

55. Wieler M, Gee M, Camicioli R, et al.: Freezing of gait in early Parkinson's disease: Nigral iron content estimated from magnetic resonance imaging. J Neurol Sci 2016, 361:87-91.

56. An H, Zeng X, Niu T, et al.: Quantifying iron deposition within the substantia nigra of Parkinson's disease by quantitative susceptibility mapping. J Neurol Sci 2018, 386:4652.

57. Guan X, Xuan M, Gu Q, et al.: Regionally progressive accumulation of iron in Parkinson's disease as measured by quantitative susceptibility mapping. NMR Biomed 2017, 30:e3489.

58. Acosta-Cabronero J, Cardenas-Blanco A, Betts MJ, et al.: The whole-brain pattern of magnetic susceptibility perturbations in Parkinson's disease. Brain 2017, 140:118-131.

59. Schwarz ST, Xing Y, Tomar P, et al.: In Vivo Assessment of Brainstem Depigmentation in Parkinson Disease: Potential as a Severity Marker for Multicenter Studies. Radiology 2017, 283:789-798.

60. Prasad S, Stezin A, Lenka A, et al.: Three-dimensional neuromelanin-sensitive magnetic resonance imaging of the substantia nigra in Parkinson's disease. Eur J Neurol 2018, 12:3218-3221.

61. Fabbri M, Reimão S, Carvalho M, et al.: Substantia Nigra Neuromelanin as an Imaging Biomarker of Disease Progression in Parkinson's Disease. J Parkinsons Dis 2017, 7:491501.

62. Sung $\mathrm{YH}$, Lee $\mathrm{J}$, Nam $\mathrm{Y}$, et al.: Differential involvement of nigral subregions in idiopathic parkinson's disease. Hum Brain Mapp 2018, 39:542-553.

63. Matsuura K, Maeda M, Tabei K, et al.: A longitudinal study of neuromelanin-sensitive magnetic resonance imaging in Parkinson's disease. Neurosci Lett 2016, 633:112-117.

64. Ofori E, Pasternak O, Planetta PJ, et al.: Increased free water in the substantia nigra of Parkinson's disease: a single-site and multi-site study. Neurobiol Aging 2015, 36:10971104.

** 65. Burciu RG, Ofori E, Archer DB, et al.: Progression marker of Parkinson's disease: a 4year multi-site imaging study. Brain 2017, 140:2183-2192.

It is the first (4 year) long-term longitudinal multicentre study to assess the $\mathrm{SN}$ free water as a surrogate biomarker of PD progression.

66. Ofori E, Krismer F, Burciu RG, et al.: Free water improves detection of changes in the substantia nigra in parkinsonism: A multisite study. Mov Disord 2017, 32:1457-1464.

67. Chung JW, Burciu RG, Ofori E, et al.: Parkinson's disease diffusion MRI is not affected by acute antiparkinsonian medication. NeuroImage Clin 2017, 14:417-421.

68. Ofori E, Pasternak O, Planetta PJ, et al.: Longitudinal changes in free-water within the substantia nigra of Parkinson's disease. Brain 2015, 138:2322-2331.

* 69. Marquié M, Verwer EE, Meltzer AC, et al.: Lessons learned about [F-18]-AV-1451 offtarget binding from an autopsy-confirmed Parkinson's case. Acta Neuropathol Commun 2017, 5:75. 
This is the first report descring the correllation between tau imaging and neuropathological underpinning in a autopsy-diagnosed PD patient.

70. Hansen $\mathrm{AK}$, Knudsen $\mathrm{K}$, Lillethorup $\mathrm{TP}$, et al.: In vivo imaging of neuromelanin in Parkinson's disease using 18 F-AV-1451 PET. Brain 2016, 139:2039-2049.

* 71. Kuya K, Shinohara Y, Miyoshi F, et al.: Correlation between neuromelanin-sensitive MR imaging and 123I-FP-CIT SPECT in patients with parkinsonism. Neuroradiology 2016, 58:351-356.

For the first time the authors of this paper correlated the dopaminergic denervation, assessed by DaT scans, with the progressive decrease in neuromelanin signal MRI.

72. Karimi $\mathrm{M}$, Tian $\mathrm{L}$, Brown $\mathrm{CA}$, et al.: Validation of nigrostriatal positron emission tomography measures: Critical limits. Ann Neurol 2013, 73:390-396.

73. Cheng $\mathrm{H}-\mathrm{C}$, Ulane $\mathrm{CM}$, Burke $\mathrm{RE}$ : Clinical progression in Parkinson disease and the neurobiology of axons. Ann Neurol 2010, 67:715-725.

74. Schreglmann SR, Krauss JK, Chang JW, et al.: Functional lesional neurosurgery for tremor: back to the future? J Neurol Neurosurg Psychiatry 2017, doi:10.1136/jnnp-2017316301 .

75. Alkemade A, de Hollander G, Keuken MC, et al.: Comparison of T2*-weighted and QSM contrasts in Parkinson's disease to visualize the STN with MRI. PLoS One 2017, 12:e0176130.

* 76. Akram H, Dayal V, Mahlknecht P, et al.: Connectivity derived thalamic segmentation in deep brain stimulation for tremor. NeuroImage Clin 2018, 18:130-142.

A high angular resolution diffusion-based imaging technique was proposed in this paper to support the VIM visualization for functional surgery

** 77. Ewert S, Plettig P, Li N, et al.: Toward defining deep brain stimulation targets in MNI space: A subcortical atlas based on multimodal MRI, histology and structural connectivity. Neuroimage 2018, 170:271-282.

This work provides a potential outstanding tool to improve the precision of functional surgery targeting and its effettiveness in tailoring the treatment of advanced PD.

** 78. Horn A, Reich M, Vorwerk J, et al.: Connectivity Predicts deep brain stimulation outcome in Parkinson disease. Ann Neurol 2017, 82:67-78.

This study disclosed the role of connectivity behind the STN DBS effectiveness in PD: authors proposed to consider also network nodes as possible future targets of neuromodulation.

79. Golestanirad L, Iacono MI, Keil B, et al.: Construction and modeling of a reconfigurable MRI coil for lowering SAR in patients with deep brain stimulation implants. Neuroimage 2017, 147:577-588.

80. Politis M, Sauerbier A, Loane C, et al.: Sustained striatal dopamine levels following intestinal levodopa infusions in Parkinson's disease patients. Mov Disord 2017, 32:235240.

81. Auffret M, Le Jeune F, Maurus A, et al.: Apomorphine pump in advanced Parkinson's disease: Effects on motor and nonmotor symptoms with brain metabolism correlations. J Neurol Sci 2017, 372:279-287.

* 82. Díez-Cirarda M, Ojeda N, Peña J, et al.: Increased brain connectivity and activation after cognitive rehabilitation in Parkinson's disease: a randomized controlled trial. Brain Imaging Behav 2017, 11:1640-1651.

This is the first study to report how the DTI changes after a 3 month cognitive rehabilitation protocol in PD lasts over 18 months of follow-up.

83. Díez-Cirarda M, Ojeda N, Peña J, et al.: Long-term effects of cognitive rehabilitation on brain, functional outcome and cognition in Parkinson's disease. Eur J Neurol 2018, 25:5-12.

84. Alberts JL, Phillips M, Lowe MJ, et al.: Cortical and motor responses to acute forced exercise in Parkinson's disease. Parkinsonism Relat Disord 2016, 24:56-62. 
85. Laruelle M, Wallace E, Seibyl JP, et al.: Graphical, Kinetic, and Equilibrium Analyses of in vivo [ 123 I] $\beta$-CIT Binding to Dopamine Transporters in Healthy Human Subjects. $J$ Cereb Blood Flow Metab 1994, 14:982-994.

86. Abi-dargham A, Gandelman MS, Deerausquin GA, et al.: SPECT Imaging of Dopamine Transporters in Human Brain with Iodine- 123-Fluoroalkyl Analogs of n-CIT. $J$ Nucl Med 1996, 37:1129-1134.

87. Xia C-F, Arteaga J, Chen G, et al.: [18F]T807, a novel tau positron emission tomography imaging agent for Alzheimer's disease. Alzheimer's Dement 2013, 9:666-676.

88. Hannestad J, Gallezot J-D, Planeta-Wilson B, et al.: Clinically Relevant Doses of Methylphenidate Significantly Occupy Norepinephrine Transporters in Humans In Vivo. Biol Psychiatry 2010, 68:854-860.

89. Klunk WE, Engler H, Nordberg A, et al.: Imaging brain amyloid in Alzheimer's disease with Pittsburgh Compound-B. Ann Neurol 2004, 55:306-319.

90. DaSilva J, Lourenco C, Meyer J, et al.: Imaging cAMP-specific phosphodiesterase-4 in

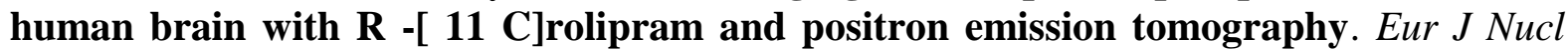
Med Mol Imaging 2002, 29:1680-1683.

91. Clark CM: Use of Florbetapir-PET for Imaging $\beta$-Amyloid Pathology. JAMA 2011, 305:275.

92. Lin K-J, Hsu W-C, Hsiao I-T, et al.: Whole-body biodistribution and brain PET imaging with [18F]AV-45, a novel amyloid imaging agent — a pilot study. Nucl Med Biol 2010, 37:497-508. 
Figure 1 - Noradrenergic system evaluated by $\left[{ }^{11} \mathrm{C}\right]$ MeNER PET. Column "A" displays tracer uptake on a section including the raphe nucleus, while columns " $\mathrm{B}$ " and "C" display sections including red nucleus, hypothalamus and thalamus. [modified from Nahimi et al.[24]]

Figure 2 - Monitoring of PD progression: SN free water content. As one of the most promising markers to quantify disease progression, SN free water has been evaluated in a longitudinal study over 4 years [modified from Burciu et al.[65**]].

Figure 3 - Comparison of STN visualization for DBS direct targeting. Examples of identifying the STN (marked by red and blue marks) by standard T2* sequences (panel A) or newly proposed QSM sequences (panel B) [modified from Alkemade et al.[75]].

Table 1: Overview of PET tracers currently used in PD imaging research. (for exhaustive list, see supplementary material in Politis' review[5]) 